\title{
A Molecular-Thermodynamic Lattice Model for Binary Mixtures*
}

\author{
Yuan Qin and John M. Prausnitz \\ Department of Chemical Engineering, University of California, Berkeley, and Chemical Sciences \\ Division, Lawrence Berkeley National Laboratory, Berkeley, CA 94720
}

\begin{abstract}
Using a method originally proposed for describing a continuum-space polymer fluid, a new expression for the Helmholtz energy of mixing is proposed for a binary lattice mixture. Molecular size asymmetry and nonrandomness due to segment-segment interactions are taken into account. An expression proposed by Yan, Liu and Hu for a binary lattice mixture of monomers, based on the Ising model, is used as a reference system. Calculated critical constants and liquid-liquid coexistence curves are in good agreement with Monte Carlo simulations for lattice mixtures with modest size asymmetry. Because lattice spacing rises with increasing temperature, comparison of calculated binary liquid-liquid equilibria with experiment requires that calculations take into account that the interchange energy falls as temperature rises. While the new expression for the Helmholtz energy of mixing provides much improvement over the Flory-Huggins equation, calculated liquid-liquid equilibria for three binary systems are similar to those from Guggenheim's quasi-chemical theory.
\end{abstract}

Keywords: lattice theory, Ising model, asymmetric mixtures, critical constants, liquid-liquid coexistence curve.

*Dedicated to the memory of Prof. Dr. E. U. Franck, a dear friend and an inspiring colleague. 


\section{Introduction}

In the early years of physical chemistry, about 120 years ago, there was much interest in the thermodynamic properties of binary liquid mixtures. Because over the years, this interest has continued, the technical literature is rich in experimental and theoretical studies of the equilibrium properties of binary liquid mixtures. Most attention has been given to the Helmholtz energy of mixing $\Delta_{\text {mix }} A$.

While many other theoretical ideas have been proposed, lattice theory provides a particularly simple method to calculate $\Delta_{\text {mix }} A[1-3]$. In this theory, each molecule occupies one or several connected sites on a three-dimensional lattice; only interactions between nearest-neighbor sites are considered. $\Delta_{\text {mix }} A$ consists of two parts, an athermal part that describes the combinatorial entropy contribution, and a residual part that is due to interactions between molecular segments. The athermal contribution to $\Delta_{\text {mix }} A$ is determined only by the shapes and sizes of the molecules and by the mixture composition. The best-known result for athermal $\Delta_{\text {mix }} A$ is given by the Flory-Huggins theory of polymer solutions $[4,5]$.

The central problem for describing residual $\Delta_{\text {mix }} A$ is to establish a relation for describing the effect of nonrandomness, that is, the tendency of molecules to show some preference in choosing their immediate neighbors. In the simplest case, for mixtures of equi-sized molecules, if we assume that there is no preference, mixing is entirely statistical; in that event, we have what is called a "regular" solution [6] with a very simple result for residual $\Delta_{\text {mix }} A$. To take nonrandomness into account, about 70 years ago, Guggenheim $[2,3,7]$ proposed a well-known approximation known as the quasi-chemical approximation. 
At normal conditions, for relatively simple mixtures where the components are (nearly) nonpolar and do not exhibit hydrogen bonding, nonrandomness has little effect on $\Delta_{\text {mix }} A$. However, if the two components are sufficiently different to show limited liquid solubility, the effect of nonrandomness becomes important, especially in the region near the upper critical temperature, that is, the temperature where the two equilibrated liquid phases become identical. To represent the liquid-liquid coexistence curve, the regular-solution theory predicts a critical temperature that is too large. The quasi-chemical theory gives better results but nevertheless, shows some deviation from experiment.

Recently, based on the Ising model, Yan, Liu and $\mathrm{Hu}$ [8] developed a new analytical theory for mixtures of equi-sized spherical molecules; this theory is better than quasi-chemical theory when compared with Monte Carlo simulations. In the present work, we generalize this theory for binary lattice mixtures containing chain molecules of different size. Toward that end, we use the thermodynamic perturbation method, originally proposed by Wertheim $[9,10]$, and by Stell and Zhou [11-13] to represent the thermodynamic properties of continuum-space polymer fluids. Compared to Freed's series-expansion theory [14] and Monte Carlo simulations [15] for lattice polymer mixtures, our generalized theory gives good agreement.

In most cases, the lattice model assumes that the volume of the mixture and the interchange energy $\varepsilon$ are independent of temperature. However, for real mixtures, calculated results must take into account that interchange energy $\varepsilon$ decreases with temperature due to expansion of the lattice. When a suitable temperature dependence for interchange energy $\varepsilon$ is assigned to each theory, our proposed theory provides much improvement over the well-known Flory-Huggins 
equation and slight improvement over Guggenheim's quasi-chemical theory.

\section{Theory}

\subsection{Theoretical framework}

For continuum-space associating fluids, Wertheim $[9,10]$ developed a thermodynamic perturbation theory. When the degree of association is unity, this theory becomes applicable to mixtures containing molecular or polymer fluids. Using a different approach, Stell and Zhou [11-13] obtained results identical to the first-order thermodynamic perturbation theory of Wertheim. Instead of deriving our theory in the customary language of a lattice model, we obtain an expression for $\Delta_{\text {mix }} A$ for a lattice mixture by comparison to that for a continuum-space polymer mixture.

We consider a binary associating-fluid mixture which contains $r_{1} N_{1}$ associating spheres 1 and $r_{2} N_{2}$ associating spheres 2. Each kind of associating sphere can react to form homogeneous polymer molecules with $r_{1}$ segments and $r_{2}$ segments, respectively. The numbers of chemical bonds of the two polymer molecules are $b_{1}$ and $b_{2}$, respectively. The shapes of the polymer molecules can be linear or branched. For molecules without rings, $b_{i}=r_{i}-1$. When the degree of association is zero, all spheres are non-bonded; we use this simple-fluid mixture as our reference fluid. When the degree of association is unity, the associating fluid becomes a binary polymer mixture which includes $N_{1}$ polymer molecules 1 and $N_{2}$ polymer molecules 2 . According to the first-order thermodynamic perturbation theory [9-13], the Helmholtz energy of the binary polymer mixture consists of three terms 


$$
A=A^{\text {id }}+A^{\text {ref }}+A^{\text {assoc }}
$$

where $A^{\text {id }}$ is the ideal contribution; $A^{\text {ref }}$ is the Helmholtz energy of the reference system in excess over the ideal part; and $A^{a s s o c}$ is the association contribution given by

$$
A^{\mathrm{assoc}}=-N_{1} b_{1} k_{\mathrm{B}} T \int_{\alpha_{1}=0}^{\alpha_{1}=1} \ln y_{11} d \alpha_{1}-N_{2} b_{2} k_{B} T \int_{\alpha_{2}=0}^{\alpha_{2}=1} \ln y_{22} d \alpha_{2}
$$

where $k_{\mathrm{B}}$ is Boltzmann's constant, $T$ is absolute temperature, $\alpha_{i}$ is the degree of association for component $i, y_{i i}$ is the cavity-correlation function for the associating spheres at the bonding distance. The cavity correlation function for the simple fluid is defined by [16]

$$
y_{i i}(r)=g_{i i}(r) \exp \left[\Gamma_{i i}(r) / k_{B} T\right]
$$

where $g_{i i}(r)$ is the radial distribution function and $\Gamma_{i i}(r)$ is the two-body potential of the non-bonded molecules.

Now we consider a binary lattice mixture. The total number of lattice sites is $N_{s}$ and the coordination number is $z$. Each site of the lattice is occupied by a molecule or a molecular segment of type 1 or 2 . Because all lattice sites are occupied

$$
N_{s}=r_{1} N_{1}+r_{2} N_{2}
$$

The volume fractions of the two components are defined by

$$
\begin{aligned}
& \phi_{1}=\frac{r_{1} N_{1}}{N_{s}} \\
& \phi_{2}=\frac{r_{2} N_{2}}{N_{s}} .
\end{aligned}
$$

For a binary mixture, there are three kinds of nearest-neighbors: 1-1, 2-2 and 1-2. The interaction energies of the three kinds of pairs are $-\varepsilon_{11},-\varepsilon_{22}$ and $-\varepsilon_{12}$, respectively. The interchange 
energy $\varepsilon$ is defined by

$$
\varepsilon=\varepsilon_{11}+\varepsilon_{22}-2 \varepsilon_{12}
$$

Although there are three energy parameters, for calculating $\Delta_{\text {mix }} A$, we require only interchange energy $\varepsilon$.

We use Eq. (1) for the binary lattice mixture but, unlike previous work, the reference system is now described by the Ising model, i.e., the binary lattice mixture of two kinds of monomers. To apply continuum-space theory to a lattice model, Eq. (2) needs some modification. Toward that end, we introduce local composition $x_{i j}^{\prime}$ of component $j$ around component $i$; local composition means that, on the average, we find $z x_{i j}^{\prime}$ nearest-neighbor segments $j$ around an arbitrarily chosen segment $i$. The local compositions satisfy the conservation equations

$$
\begin{aligned}
& x_{11}^{\prime}+x_{12}^{\prime}=1 \\
& x_{21}^{\prime}+x_{22}^{\prime}=1 .
\end{aligned}
$$

In continuum space, $\rho_{j} g_{i j}(r) 4 \pi r^{2} d r$ is the average number of segments $j$ at distance $r$ given that one segment of component $i$ is at the origin, where $\rho_{j}$ is the number density of segments $j$. Because $z x_{i j}^{\prime}$ and $\rho_{j} g_{i j}(r) 4 \pi r^{2} d r$ have similar physical meaning, the "radial distribution function" for a lattice mixture is $x_{i j}^{\prime} / x_{j}^{\prime}$, where $x_{j}^{\prime}$ is the bulk composition of component $j$. Thus the "cavity-correlation function" for a lattice mixture is $y_{i j}=\exp \left(-\varepsilon_{i j} / k_{B} T\right) x_{i j}^{\prime} / x_{j}^{\prime}$. According to this analogy, for a binary lattice mixture, the association Helmholtz energy in Eq. (2) becomes

$$
A^{\text {assoc }}=-N_{1} b_{1} k_{B} T \int_{\alpha_{1}=0}^{\alpha_{1}=1} \ln \frac{\exp \left(-\varepsilon_{11} / k_{B} T\right) x_{11}^{\prime}}{x_{1}^{\prime}} d \alpha_{1}-N_{2} b_{2} k_{B} T \int_{\alpha_{2}=0}^{\alpha_{2}=1} \ln \frac{\exp \left(-\varepsilon_{22} / k_{B} T\right) x_{22}^{\prime}}{x_{2}^{\prime}} d \alpha_{2} .
$$




\subsection{Athermal mixture}

The number of possible states for an ideal lattice mixture is

$$
\Omega=\frac{N_{s}^{N_{1}+N_{2}}}{N_{1} ! N_{2} !} f_{1}^{N_{1}} f_{2}^{N_{2}}
$$

where $f_{1}$ and $f_{2}$ are the possible configuration numbers of the two kinds of molecules. The number of possible states for the pure lattice fluid is

$$
\Omega_{i}=\frac{\left(N_{i} r_{i}\right)^{N_{i}}}{N_{i} !} f_{i}^{N_{i}} .
$$

Then, the ideal contribution to the Helmholtz energy of mixing is

$$
\begin{aligned}
\frac{\Delta_{\text {mix }} A^{\text {id }}}{N_{s} k_{B} T} & =\frac{A^{\text {id }}}{N_{s} k_{B} T}-\frac{A_{1}^{\text {id }}}{N_{s} k_{B} T}-\frac{A_{2}^{\text {id }}}{N_{s} k_{B} T} \\
& =\frac{1}{N_{s}}\left(-\ln \Omega+\ln \Omega_{1}+\ln \Omega_{2}\right) \\
& =\frac{\phi_{1}}{r_{1}} \ln \phi_{1}+\frac{\phi_{2}}{r_{2}} \ln \phi_{2} .
\end{aligned}
$$

Eq. (13) is identical to the equation obtained by Flory [4] more than 60 years ago, using an entirely different derivation.

For an athermal lattice mixture, the reference system is an athermal mixture of monomers.

From the combinatorial argument, the total Helmholtz energy of mixing for the athermal reference system is

$$
\frac{\Delta_{\text {mix }} A}{N_{s} k_{B} T}=\phi_{1} \ln \phi_{1}+\phi_{2} \ln \phi_{2}
$$

However, according to Eq. (13), when $r_{1}=r_{2}=1$, the ideal part of $\Delta_{\text {mix }} A / N_{s} k_{B} T$ for the 
reference system is also $\phi_{1} \ln \phi_{1}+\phi_{2} \ln \phi_{2}$. Because in Eq. (2) the reference system contribution to the Helmholtz energy of mixing is the difference between the two terms,

$$
\frac{\Delta_{\text {mix }} A^{\text {ref,athermal }}}{N_{s} k_{\mathrm{B}} T}=0 .
$$

For calculating the association Helmholtz energy for the athermal system, we introduce another molecular parameter: the surface of molecule $i$, viz $z q_{i}$

$$
z q_{i}=z r_{i}-2 b_{i}
$$

The surface fractions are defined by

$$
\begin{aligned}
& \theta_{1}=\frac{q_{1} N_{1}}{q_{1} N_{1}+q_{2} N_{2}} \\
& \theta_{2}=\frac{q_{2} N_{2}}{q_{1} N_{1}+q_{2} N_{2}} .
\end{aligned}
$$

We assume that, for the athermal mixture, the contacting pairs distribute randomly. We consider that first, component 1 associates to form polymers while component 2 consists of monomers. When the degree of association is $\alpha_{1}$, the molecular surface of the monomers of component 1 is $N_{s} \phi_{1}\left(1-\alpha_{1}\right) z$. The total molecular surface of the mixture is $N_{s}\left[\phi_{1} \alpha_{1} q_{1} z+\phi_{1}\left(1-\alpha_{1}\right) z+\phi_{2} z\right]$. If the contacting pairs distribute randomly, then

$$
\begin{aligned}
\frac{x_{11}^{\prime}}{x_{1}^{\prime}} & =\frac{\phi_{1}\left(1-\alpha_{1}\right) z}{\phi_{1} \alpha_{1} q_{1} z+\phi_{1}\left(1-\alpha_{1}\right) z+\phi_{2} z} \cdot \frac{1}{\phi_{1}\left(1-\alpha_{1}\right)} \\
& =\frac{1}{\phi_{1} \alpha_{1} q_{1}+\phi_{1}\left(1-\alpha_{1}\right)+\phi_{2}} .
\end{aligned}
$$

Next, we consider the case where component 1 is completely associated and the degree of association of component 2 is $\alpha_{2}$. Then 


$$
\begin{aligned}
\frac{x_{22}^{\prime}}{x_{2}^{\prime}} & =\frac{\phi_{2}\left(1-\alpha_{2}\right) z}{\phi_{1} q_{1} z+\phi_{2} \alpha_{2} q_{2} z+\phi_{2}\left(1-\alpha_{2}\right) z} \cdot \frac{1}{\phi_{2}\left(1-\alpha_{2}\right)} \\
& =\frac{1}{\phi_{1} q_{1}+\phi_{2} \alpha_{2} q_{2}+\phi_{2}\left(1-\alpha_{2}\right)} .
\end{aligned}
$$

According to Eq (11), the association athermal Helmholtz energy for a binary mixture and that for a pure fluid $i$ are, respectively

$$
\begin{gathered}
\frac{A_{\text {mixture }}^{\text {assoc,athermal }}}{k_{\mathrm{B}} T}=\frac{z}{2}\left(N_{1} q_{1} \ln \frac{\theta_{1} r_{1}}{\phi_{1}}+N_{2} q_{2} \ln \frac{\theta_{2} r_{2}}{\phi_{2}}\right) \\
\frac{A_{i}^{\text {assoc,athermal }}}{k_{\mathrm{B}} T}=\frac{z}{2} N_{i} q_{i} \ln r_{i} .
\end{gathered}
$$

The Helmholtz energy of mixing for an athermal mixture is

$$
\begin{aligned}
\frac{\Delta_{\text {mix }} A^{\text {athermal }}}{N_{s} k T} & =\frac{A_{\text {mixture }}^{\text {athermal }}}{N_{s} k T}-\frac{A_{1}^{\text {athermal }}}{N_{s} k T}-\frac{A_{2}^{\text {athermal }}}{N_{s} k T} \\
& =\frac{\phi_{1}}{r_{1}} \ln \phi_{1}+\frac{\phi_{2}}{r_{2}} \ln \phi_{2}+\frac{z}{2}\left(\frac{\phi_{1} q_{1}}{r_{1}} \ln \frac{\theta_{1}}{\phi_{1}}+\frac{\phi_{2} q_{2}}{r_{2}} \ln \frac{\theta_{2}}{\phi_{2}}\right) .
\end{aligned}
$$

Many years ago, Staverman [17] obtained the same result using an entirely different derivation.

\subsection{Non-athermal mixture}

The Flory-Huggins theory uses Eq. (13) for the athermal part and a mean-field term for the residual part. The total Helmholtz energy of mixing is

$$
\frac{\Delta_{\text {mix }} A}{N_{s} k T}=\frac{\phi_{1}}{r_{1}} \ln \phi_{1}+\frac{\phi_{2}}{r_{2}} \ln \phi_{2}+\frac{z}{2} \phi_{1} \phi_{2} \varepsilon
$$

Eq. (24) is the well-known Flory-Huggins equation [4].

For the quasi-chemical theory of Guggenheim[2, 3, 7], the Helmholtz energy of mixing is 


$$
\begin{aligned}
\frac{\Delta_{\text {mix }} A}{N_{s} k T}= & \frac{\phi_{1}}{r_{1}} \ln \phi_{1}+\frac{\phi_{2}}{r_{2}} \ln \phi_{2}+\frac{z}{2}\left(\frac{\phi_{1} q_{1}}{r_{1}} \ln \frac{\theta_{1}}{\phi_{1}}+\frac{\phi_{2} q_{2}}{r_{2}} \ln \frac{\theta_{2}}{\phi_{2}}\right) \\
& +\frac{z}{2}\left[\phi_{1} \ln \frac{\beta-1+2 \phi_{1}}{\phi_{1}(\beta+1)}+\phi_{2} \ln \frac{\beta-1+2 \phi_{2}}{\phi_{2}(\beta+1)}\right]
\end{aligned}
$$

with

$$
\begin{aligned}
& \beta=\left(1+4 \phi_{1} \phi_{2} C\right)^{1 / 2} \\
& C=\exp (\varepsilon / k T)-1 .
\end{aligned}
$$

For a binary non-athermal mixture, we use the Ising model for our reference system. Recently, Yan, Liu and $\mathrm{Hu}$ [8] proposed a new approximation for the Ising model for a mixture of equi-sized molecules with $r_{1}=r_{2}=1$. They find that the local compositions of the Ising model satisfy

$$
\begin{aligned}
& \frac{x_{11}^{\prime}}{x_{12}^{\prime}}=\frac{\phi_{1}}{\phi_{2}}\left(1+\phi_{2} C\right) \\
& \frac{x_{22}^{\prime}}{x_{21}^{\prime}}=\frac{\phi_{2}}{\phi_{1}}\left(1+\phi_{1} C\right) .
\end{aligned}
$$

From Eqs. (24) and (25), Yan, Liu and Hu [8] obtain the residual Helmholtz energy of mixing

$$
\frac{\Delta_{\text {mix }} A^{\text {ref,residual }}}{N_{s} k T}=\frac{z \phi_{1} \phi_{2}}{2\left(1-\phi_{1} \phi_{2}\right)}\left[\frac{\varepsilon}{k T}-\ln \left(1+\phi_{1} \phi_{2} C\right)\right] .
$$

Because Yan et al. considered only mixtures of equi-sized spherical molecules, their expressions use mole fractions rather than volume fractions. We use volume fractions here because we are interested in asymmetric mixtures. From Eqs. (8), (9), (28) and (29), we also obtain

$$
\frac{x_{11}^{\prime}}{x_{1}^{\prime}}=\frac{1+\phi_{2} C}{1+\phi_{1} \phi_{2} C}
$$




$$
\frac{x_{22}^{\prime}}{x_{2}^{\prime}}=\frac{1+\phi_{1} C}{1+\phi_{1} \phi_{2} C}
$$

Substituting Eqs. (31), (32) into Eq. (11), we obtain the contribution of association to the residual Helmholtz energy of mixing

$$
\begin{aligned}
\frac{\Delta_{\text {mix }} A^{\text {assoc,residual }}}{N_{s} k T} & =\frac{A^{\text {assoc,residual }}}{N_{s} k T}-\frac{A_{1}^{\text {assoc,residual }}}{N_{s} k T}-\frac{A_{2}^{\text {assoc,residual }}}{N_{s} k T} \\
& =-\frac{b_{1} \phi_{1}}{r_{1}} \ln \frac{1+\phi_{2} C}{1+\phi_{1} \phi_{2} C}-\frac{b_{2} \phi_{2}}{r_{2}} \ln \frac{1+\phi_{1} C}{1+\phi_{1} \phi_{2} C} .
\end{aligned}
$$

Combining Eqs. (30) and (33), we obtain the total residual Helmholtz energy of mixing

$$
\frac{\Delta_{\text {mix }} A^{\text {residual }}}{N_{s} k T}=\frac{z \phi_{1} \phi_{2}}{2\left(1-\phi_{1} \phi_{2}\right)}\left[\frac{\varepsilon}{k T}-\ln \left(1+\phi_{1} \phi_{2} C\right)\right]-\frac{b_{1} \phi_{1}}{r_{1}} \ln \frac{1+\phi_{2} C}{1+\phi_{1} \phi_{2} C}-\frac{b_{2} \phi_{2}}{r_{2}} \ln \frac{1+\phi_{1} C}{1+\phi_{1} \phi_{2} C}
$$

Upon adding Eq. (34) to Eq. (23), we finally obtain the total Helmholtz energy of mixing for a binary non-athermal mixture

$$
\begin{aligned}
\frac{\Delta_{\text {mix }} A}{N_{s} k T}= & \frac{\phi_{1}}{r_{1}} \ln \phi_{1}+\frac{\phi_{2}}{r_{2}} \ln \phi_{2}+\frac{z}{2}\left(\frac{\phi_{1} q_{1}}{r_{1}} \ln \frac{\theta_{1}}{\phi_{1}}+\frac{\phi_{2} q_{2}}{r_{2}} \ln \frac{\theta_{2}}{\phi_{2}}\right) \\
& +\frac{z \phi_{1} \phi_{2}}{2\left(1-\phi_{1} \phi_{2}\right)}\left[\frac{\varepsilon}{k T}-\ln \left(1+\phi_{1} \phi_{2} C\right)\right]-\frac{b_{1} \phi_{1}}{r_{1}} \ln \frac{1+\phi_{2} C}{1+\phi_{1} \phi_{2} C}-\frac{b_{2} \phi_{2}}{r_{2}} \ln \frac{1+\phi_{1} C}{1+\phi_{1} \phi_{2} C}
\end{aligned}
$$

\section{Comparison with series -expansion theory and computer simulations}

We now compare Eq. (35) with Freed's results from a series expansion [14] and with results from Monte Carlo simulations [15] for a lattice mixture.

\subsection{Comparison with Freed's series -expansion theory}

Bawendi and Freed [14] developed an exact series-expansion theory for lattice mixtures. For 
the athermal contribution to $\Delta_{\text {mix }} A$, this theory gives

$$
\begin{aligned}
\frac{\Delta_{\text {mix }} A^{\text {athermal }}}{N_{s} k T} & =\frac{\phi_{1}}{r_{1}} \ln \phi_{1}+\frac{\phi_{2}}{r_{2}} \ln \phi_{2}+\left(\frac{1}{r_{1}}-\frac{1}{r_{2}}\right)^{2} \phi_{1} \phi_{2} \frac{1}{z}-\left\{8+\frac{1}{r_{1} r_{2}}\left[\frac{2}{3}\left(6-11 r_{1}-22 r_{2}\right)\right]\right. \\
& -\frac{2}{r_{1}^{2} r_{2}^{2}}\left[\left(r_{1}^{2}+2 r_{1} r_{2}-4 r_{1}^{2} r_{2}+3 r_{2}^{2}-8 r_{1} r_{2}^{2}+6 r_{1}^{2} r_{2}^{2}\right) \phi_{1}^{2}+\left(r_{1}^{2}+2 r_{1} r_{2}-4 r_{1}^{2} r_{2}+3 r_{2}^{2}-8 r_{1} r_{2}^{2}+6 r_{1}^{2} r_{2}^{2}\right) \phi_{2}^{2}\right. \\
& \left.\left.+3\left(2 r_{1} r_{2}-r_{1}-r_{2}\right)\right]\right\}\left(\frac{1}{r_{1}}-\frac{1}{r_{2}}\right)^{2} \phi_{1} \phi_{2} \frac{1}{z^{2}} \\
& +O\left(\frac{1}{z^{3}}\right) .
\end{aligned}
$$

To compare with Freed's result, we expand Eq. (23) in a power series of $1 / z$ to order $1 / z^{2}$ giving

$$
\begin{aligned}
\frac{\Delta_{\text {mix }} A^{\text {athermal }}}{N_{s} k T} & =\frac{\phi_{1}}{r_{1}} \ln \phi_{1}+\frac{\phi_{2}}{r_{2}} \ln \phi_{2}+\left(\frac{1}{r_{1}}-\frac{1}{r_{2}}\right)^{2} \phi_{1} \phi_{2} \frac{1}{z}-\frac{2 \phi_{1} \phi_{2}\left(r_{1}-r_{2}\right)\left(r_{1}+r_{2}-3 r_{1} r_{2}+r_{1} \phi_{1}+r_{2} \phi_{2}\right)}{3 r_{1}^{3} r_{2}^{3}} \frac{1}{z^{2}} \\
& +O\left(\frac{1}{z^{3}}\right) .
\end{aligned}
$$

For the residual Helmholtz energy of mixing, Freed's theory (and our theory) consist of two parts: a reference part, independent of the segment numbers $r_{1}$ and $r_{2}$, and an association part, that is a function of these segment numbers. For the reference system, the series-expansion of our theory is

$$
\frac{\Delta_{\text {mix }} A^{\text {ref,residual }}}{N_{s} k T}=\frac{z}{2} \frac{\varepsilon}{k T} \phi_{1} \phi_{2}-\frac{z}{4}\left(\frac{\varepsilon}{k T}\right)^{2} \phi_{1}^{2} \phi_{2}^{2}-\frac{z}{12}\left(\frac{\varepsilon}{k T}\right)^{3} \phi_{1}^{2} \phi_{2}^{2}\left(\phi_{1}^{2}+\phi_{2}^{2}\right)+O\left(\left(\frac{\varepsilon}{k T}\right)^{4}\right)
$$

while that of Freed is 


$$
\frac{\Delta_{\mathrm{mix}} A^{\text {ref,residual }}}{N_{s} k T}=\frac{z}{2} \frac{\varepsilon}{k T} \phi_{1} \phi_{2}-\frac{z}{4}\left(\frac{\varepsilon}{k T}\right)^{2} \phi_{1}^{2} \phi_{2}^{2}+O\left(\left(\frac{\varepsilon}{k T}\right)^{3}\right)
$$

For the association part, the series expansion of our theory is

$$
\frac{\Delta_{\text {mix }} A^{\text {assoc,residual }}}{N_{s} k T}=\left(-\frac{r_{1}-1}{r_{1}} \phi_{2}-\frac{r_{2}-1}{r_{2}} \phi_{1}\right) \varepsilon+O\left(\varepsilon^{2}, \varepsilon z^{-1}\right) .
$$

Freed's theory gives the same result.

Compared with Freed's series expansion, our theory is correct up to the $1 / z$ term for the athermal part; up to the $(\varepsilon / k T)^{2}$ term for the residual reference part; and up to the $\varepsilon / k T$ term for the residual association part.

\subsection{Comparison with molecular simulations}

Using Eq. (35), we calculate critical parameters for binary lattice mixtures; we compare our results with those from Monte Carlo simulations by Yan, Liu and $\mathrm{Hu}$ [15], from Flory-Huggins theory, from quasi-chemical theory and from truncated Freed theory, as shown in Fig. 1. We find that, compared to simulation results, our theory predicts somewhat larger critical temperatures and somewhat smaller critical compositions. Our theory shows much improvement over Flory-Huggins theory, and some improvement over quasi-chemical theory and over truncated Freed theory.

Using an expanded scale, Fig. 2 shows calculated liquid-liquid coexistence curves for two binary mixtures. As expected, the Flory-Huggins equation gives poor results. For the simple mixture $\left(r_{1}=r_{2}=1\right)$, agreement is very good. For the other mixture $\left(r_{1}=4, r_{2}=1\right)$ calculated critical temperatures are too large. However, results from our theory are somewhat better than 
those from quasi-chemical theory.

\section{Comparison with experiment}

We now compare results from Eq. (35) for vapor-liquid equilibria (VLE) and liquid-liquid equilibria (LLE) with those from experimental data. We assume that the coordination number $z$ is 10 , the number of bonds $b_{i}=r_{i}-1$, and the surface of the molecules $z_{i} q_{i}$ can be calculated from Eq. (16). The number of molecular segments is proportional to the molar volume of the pure liquid $v_{m, i}$. We designate the large molecules as component 1 , and the small ones as component 2 . We set $r_{2}=1$. Therefore, $r_{1}=v_{m, 1} / v_{m, 2}$.

However, unlike previous work, we consider the effect of temperature on interchange energy $\varepsilon$. We call attention to the oft-neglected fact that, while $\varepsilon$ does not directly depend on temperature; it does so indirectly because, as temperature rises, lattice spacing increases. When the distance between closest neighbors increases, we expect the absolute values of $\varepsilon_{11}, \varepsilon_{22}$ and $\varepsilon_{12}$ to decrease. If this decrease is similar for all these energies, the absolute value of $\varepsilon$ falls as temperature rises. We designate the lattice site-site separation by $L$. Then the total volume $V=N_{s} L^{3}$. From the London theory for attractive forces, we assume that the interchange energy depends on $L$ according to

$$
\begin{aligned}
\varepsilon & =-\left(\varepsilon_{11,0}+\varepsilon_{22,0}-2 \varepsilon_{12,0}\right) \frac{1}{L^{6}} \\
& =-\varepsilon_{0} \frac{1}{L^{6}}
\end{aligned}
$$

where $\varepsilon_{0}$ is a constant independent of $L$. Then, 


$$
\begin{aligned}
& \left(\frac{\partial \varepsilon}{\partial T}\right)_{P}=\left(\frac{\partial \varepsilon}{\partial L}\right)_{P}\left(\frac{\partial L}{\partial V}\right)_{P}\left(\frac{\partial V}{\partial T}\right)_{P} \\
& =\left(6 \varepsilon_{0} \frac{1}{L^{7}}\right)\left(\frac{1}{3 L^{2} N_{s}}\right)\left(\frac{\partial V}{\partial T}\right)_{P} \\
& =-2 \varepsilon \frac{1}{V}\left(\frac{\partial V}{\partial T}\right)_{P} \\
& =-2 \varepsilon \alpha_{T}
\end{aligned}
$$

or

$$
\left(\frac{\partial \ln \varepsilon}{\partial T}\right)_{P}=-2 \alpha_{T}
$$

where $\alpha_{T}=(\partial V / \partial T)_{P} / V$ is the coefficient of thermal expansion of the mixture. If we assume that $\alpha_{T}$ is a constant, then $\ln \varepsilon$ is a linear function of temperature.

Using our theory, we compare calculated isothermal VLE with experiment for two simple liquid systems: isooctane / carbon tetrachloride [18] and isooctane / benzene [19]. Tables 1 and 2 show the pertinent molecular parameters obtained from experimental VLE data. For these simple systems, as expected, we obtain very good VLE representation as shown in Figs. 3 and 4. For two temperatures, we also show calculated and experimental excess Helmholtz energy $\Delta_{\text {mix }} A^{\mathrm{E}}$ in Figs. 5 and 6. Fig. 7 shows the effect of temperature on interchange energy $\varepsilon$.

A much more stringent test of our theory is provided by its ability to represent the coexistence curve for LLE. We consider the nearly symmetric $\mathrm{CF}_{4} / \mathrm{CH}_{4}$ system [20-21]. For the temperature dependence of $\varepsilon$, we interpolate using experimental data well below and somewhat above the upper critical solution temperature as indicated in Table 3. Fig. 8 compares experimental results with those calculated with the Flory-Huggins theory, with the quasi-chemical theory and with Eq. (35). The upper critical temperature calculated with the Flory-Huggins theory 
is excessively high, as expected. The quasi-chemical theory provides much improvement. Results from our theory are slightly better than those from quasi-chemical theory.

Finally, we consider LLE for two binary systems with appreciable size asymmetry. For the two binary systems $\left(\mathrm{C}_{3} \mathrm{~F}_{7} \mathrm{COOCH}_{2}\right)_{4} \mathrm{C} / \mathrm{CHCl}_{3}$ and $\left(\mathrm{C}_{3} \mathrm{~F}_{7} \mathrm{COOCH}_{2}\right)_{4} \mathrm{C} / \mathrm{CCl}_{4}$, Fig. 9 compares experimental results [22] with those calculated using Eq. (35) and those from quasi-chemical theory. Again, our theory provides only slight improvement over the quasi-chemical theory. It is likely that the very similar results from our theory and those from quasi-chemical theory are a direct consequence of allowing interchange energy $\varepsilon$ to vary with temperature, as indicated in the caption for Fig. 9.

\section{Conclusion}

Using lattice theory and a generalization of an expression by Yan, Liu and $\mathrm{Hu}$ based on the Ising model for mixtures of equi-sized molecules, we have established a new analytic equation for the Helmholtz energy of mixing two liquids composed of molecules that differ in size. Our equation provides an approximation to describe the effect of nonrandom mixing. For the relatively simple mixtures discussed here, this effect is negligible in vapor-liquid equilibria remote from critical conditions, but it is important for liquid-liquid equilibria, especially near the upper critical temperature. When compared with molecular simulations (where interchanger energy $\varepsilon$ is independent of temperature), our theory gives good results; but when compared with experimented liquid-liquid data, our theory shows little improvement over those obtained from quasi-chemical theory. The similarity of results from these two theories follows because, for each 
theory, we have taken into account that interchange energy $\varepsilon$ depends on temperature due to expansion of the lattice as temperature rises.

\section{Acknowledgment}

For financial support, the authors are grateful to the Office for Basic Sciences of the US Dept.

of Energy. 


\section{References}

1. J. A. Barker, Lattice Theories of the Liquid State, Pergamon Press, Oxford (1963).

2. E. A. Guggenheim, Mixtures, Oxford University Press, Oxford (1952).

3. E. A. Guggenheim, Applications of Statistical Mechanics, Oxford University Press, Oxford (1966).

4. P. J. Flory, J. Chem. Phys. 10 (1942) 51.

5. M. L. Huggins, Ann. New York Acad. Sci. 43 (1942) 9.

6. J. H. Hildebrand, J. M. Prausnitz and R. L. Scott, Regular and Related Solutions, Van Nostrand Reinhold Co., New York (1970).

7. E. A. Guggenheim, Proc. Roy. Soc. A 148 (1935) 304.

8. Q. L. Yan, H. L. Liu and Y. Hu, Fluid Phase Equilibria 218 (2004) 157.

9. M. S. Wertheim, J. Stat. Phys. 35 (1984) 19, 35 (1984) 35, 42 (1986) 459, 42 (1986) 477.

10. M. S. Wertheim, J. Chem. Phys. 85 (1986) 2929.

11. G. Stell and Y. Q. Zhou, J. Chem. Phys. 91 (1989) 3618.

12. Y. Q. Zhou and G. Stell, J. Chem. Phys. 96 (1992) 1504.

13. Y. Q. Zhou and G. Stell, J. Chem. Phys. 96 (1992) 1507.

14. M. G. Bawendi and K. F. Freed, J. Chem. Phys. 88 (1988) 2741.

15. Q. L. Yan, H. L. Liu and Y. Hu, Macromolecules. 29 (1996) 4066.

16. D. Chandler, Introduction to Modern Statistical Mechanics. Oxford University Press, New York (1987), page 213. 
17. A. J. Staverman, Rec. Trav. Chim. Pays-bas. 69 (1950) 163.

18. R. Battino, J. Phys. Chem. 72 (1968) 4503.

19. S. Weissman and S. E. Wood, J. Chem. Phys. 32 (1960) 1153.

20. N. Thorp and R. L. Scott, J. Phys. Chem. 60 (1956) 670.

21. I. M. Croll and R. L. Scott, J. Phys. Chem. 62 (1958) 1441.

22. K. Shinoda and J. H. Hildebrand, J. Phys. Chem. 62 (1958) 481. 
Table 1. Molecular parameters from VLE data for the isooctane (1) / carbon tetrachloride (2) system; $r_{1}=1.71, r_{2}=1.00$.

\begin{tabular}{ccc}
\hline$T / \mathrm{K}$ & $\varepsilon / k_{B} T$ & $\ln \left(\varepsilon / k_{B} / \mathrm{K}\right)$ \\
\hline 308 & 18.1 & 2.90 \\
318 & 17.5 & 2.86 \\
328 & 17.0 & 2.83 \\
338 & 16.4 & 2.80 \\
348 & 15.9 & 2.77 \\
\hline
\end{tabular}

Table2. Molecular parameters from VLE data for the isooctane (1) / benzene (2) system; $r_{1}=1.86, r_{2}=1.00$.

\begin{tabular}{ccc}
\hline$T / \mathrm{K}$ & $\varepsilon / k_{B} T$ & $\ln \left(\varepsilon / k_{B} / \mathrm{K}\right)$ \\
\hline 308 & 37.9 & 3.63 \\
318 & 36.3 & 3.59 \\
328 & 34.9 & 3.55 \\
338 & 33.7 & 3.52 \\
348 & 32.7 & 3.49 \\
\hline
\end{tabular}


Table 3. The interchange energy, $\varepsilon / k_{B} / \mathrm{K}$, obtained from VLE and LLE data for the $\mathrm{CF}_{4}(1) /$ $\mathrm{CH}_{4}(2)$ system by different theories; $r_{1}=1.32, r_{2}=1.00$.

\begin{tabular}{cccc}
\hline$T / \mathrm{K}$ & Flory-Huggins & Quasi-chemical & Eq. (35) \\
\hline 88.0 & 38.6 & 42.4 & 42.0 \\
105.5 & 33.8 & 36.0 & 36.2 \\
108.5 & 31.8 & 33.7 & 33.9 \\
110.5 & 31.3 & 33.1 & 33.3 \\
\hline
\end{tabular}



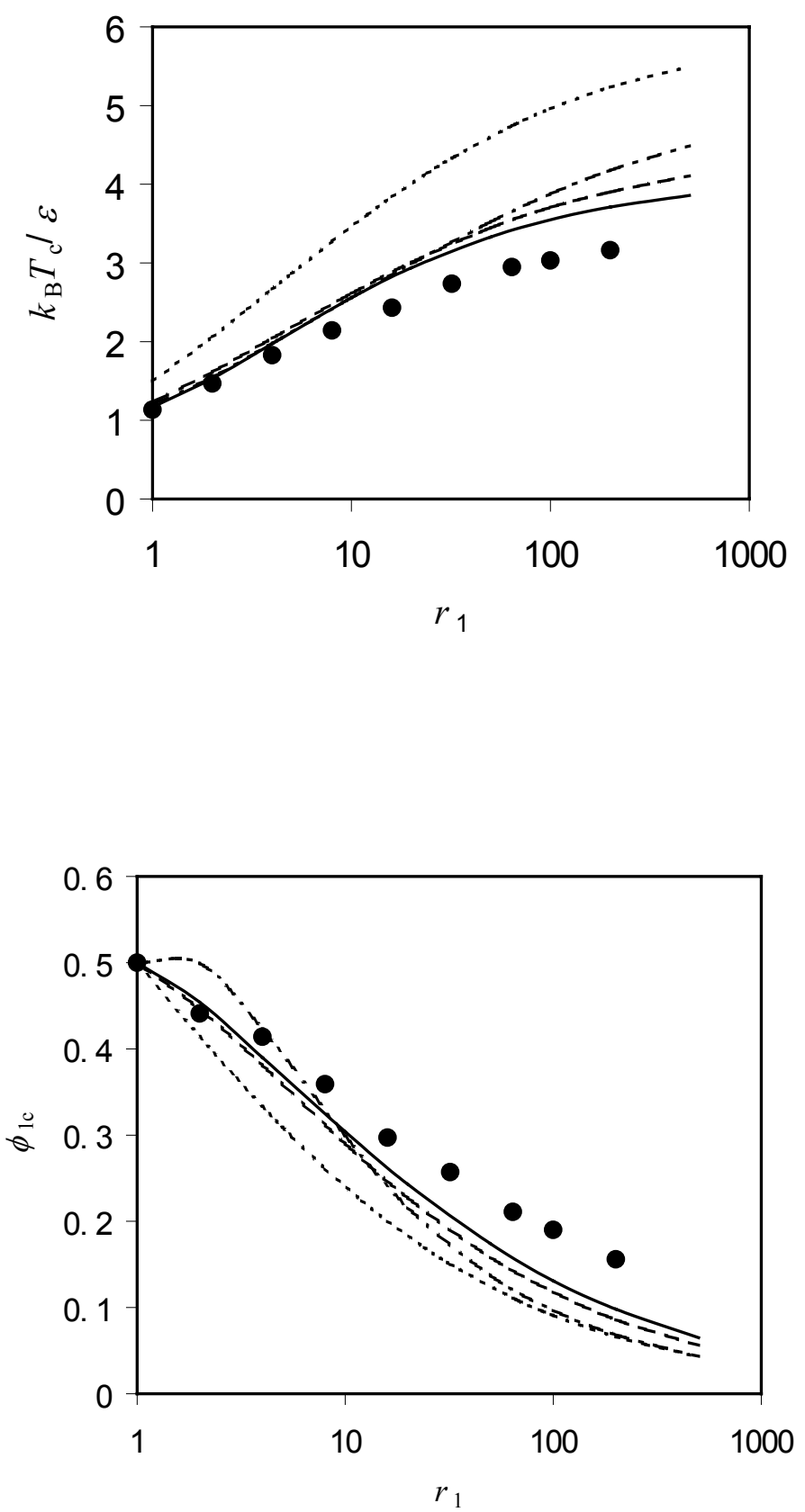

Fig. 1. Critical temperature $T_{c}$ and critical volume fraction $\phi_{1 c}$ for binary lattice mixtures as a function of polymer segment number $r_{1}$. Lattice coordination number is 6 and $r_{2}=1$. Dot: Monte Carlo simulation [15]; Dotted line: Flory-Huggins equation; Dashed line: Quasi-chemical equation; Dashed and dotted line: Freed theory; Solid line: Eq. (35). 


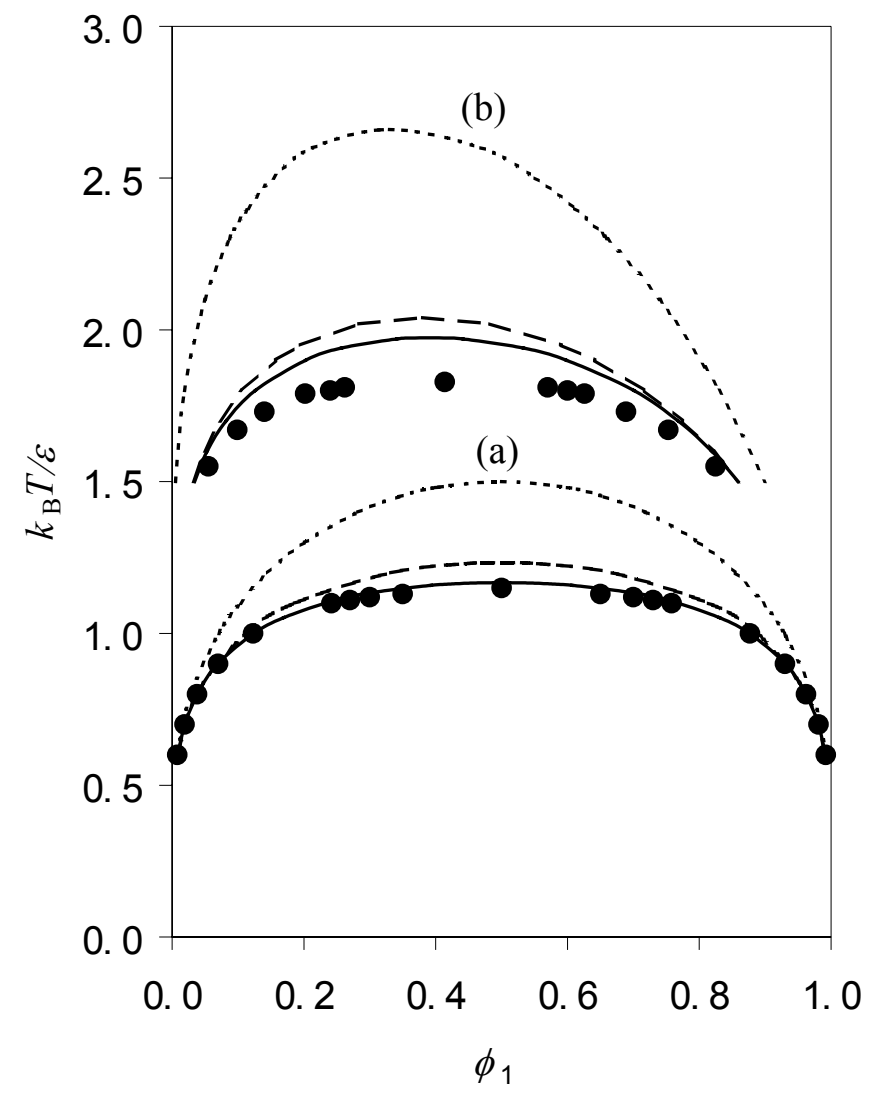

Fig. 2. Liquid-liquid equilibria for two lattice binary mixtures. Lattice coordination number is 6; interchange energy $\varepsilon$ is independent of temperature. (a): $r_{1}=1, r_{2}=1 ;(b): r_{1}=4, r_{2}=1$. Dot: Monte Carlo simulation [15]; Dotted line: Flory-Huggins theory; Dashed line: Quasi-chemical theory; Solid line: Eq. (35). 


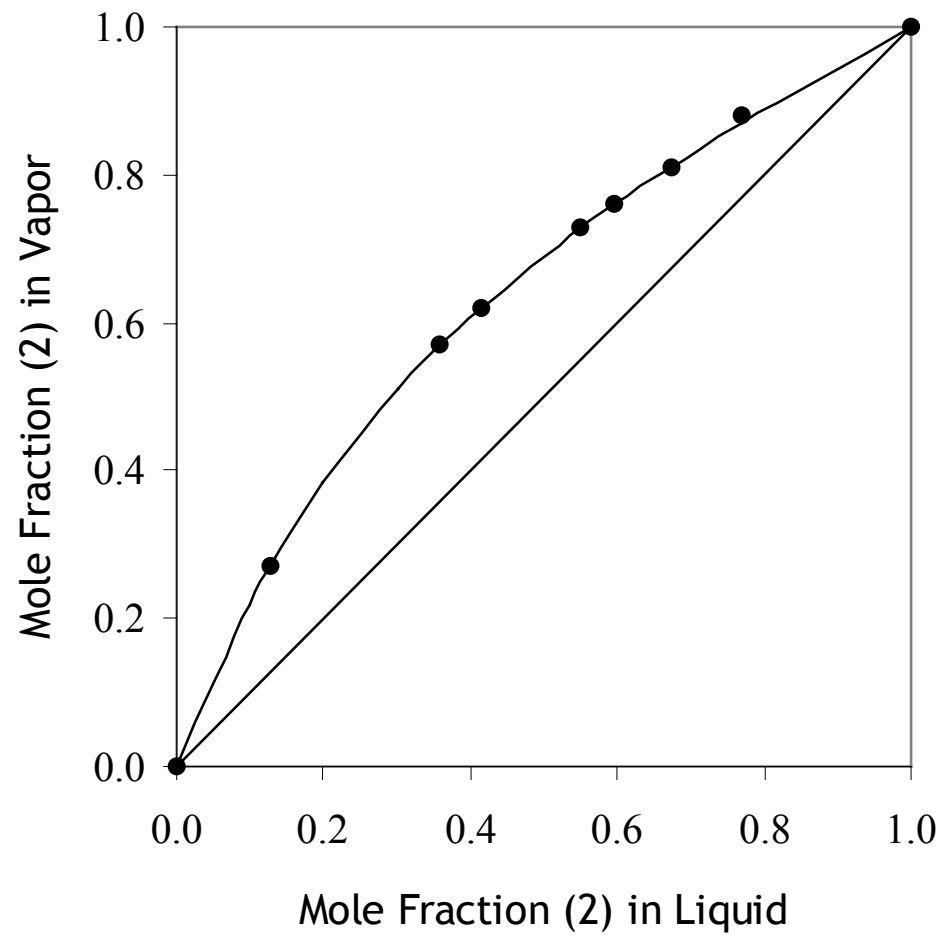

Fig. 3. Vapor-liquid equilibria for the isooctane (1) / carbon tetrachloride (2) system at $308 \mathrm{~K}$. 


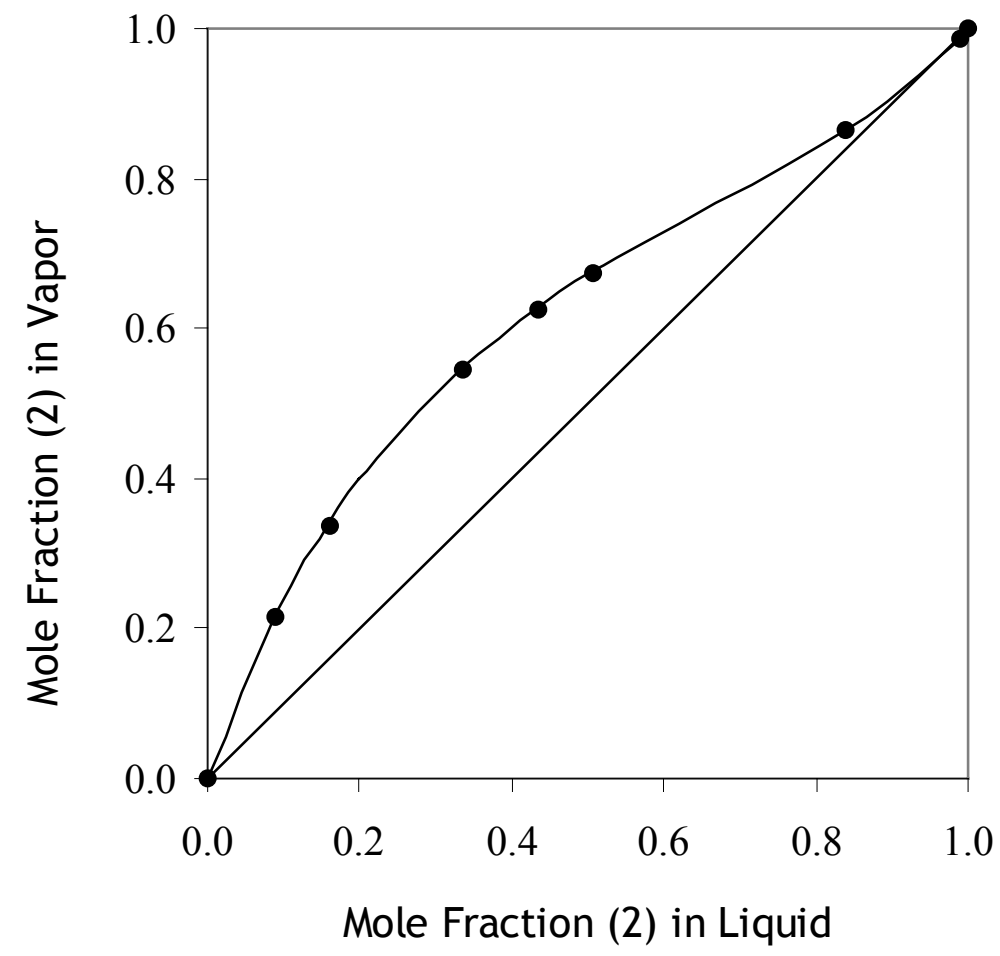

Fig. 4. Vapor-liquid equilibria for the isooctane (1) / benzene (2) system at $308 \mathrm{~K}$. 


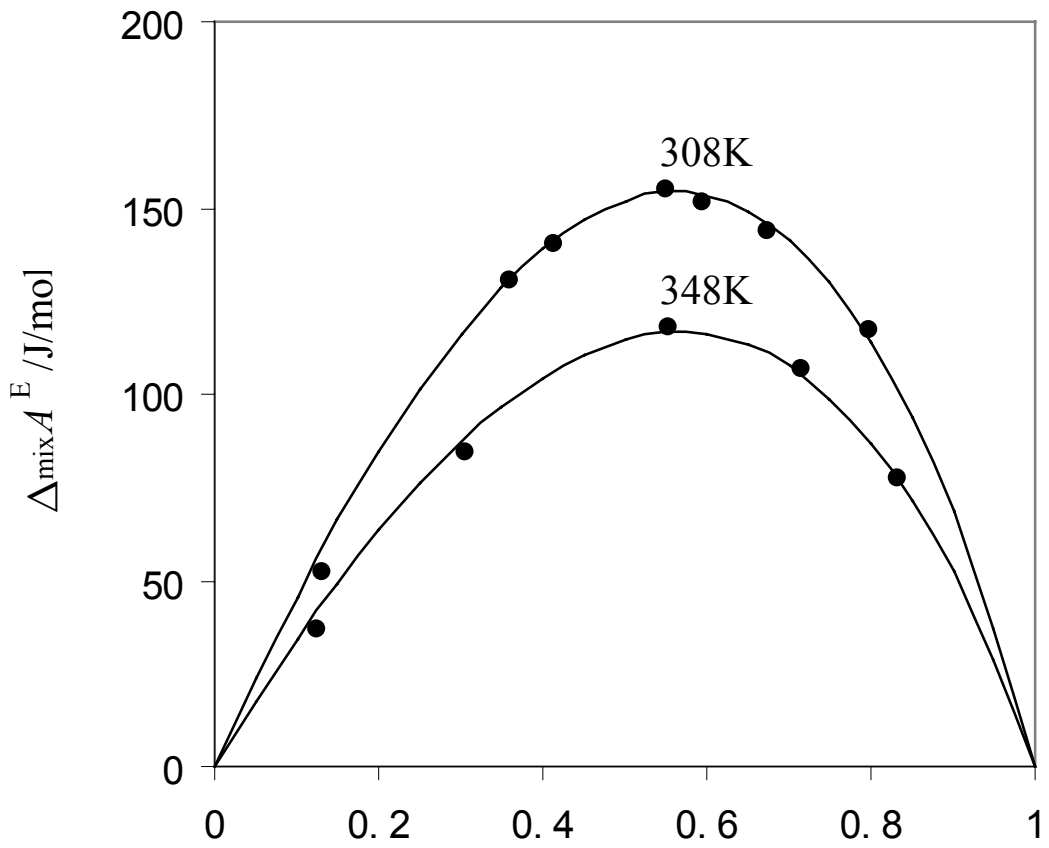

Mole Fraction of Component 2

Fig. 5. Excess Helmholtz energies for the isooctane (1) / carbon tetrachloride (2) system at 308K and 348K. Dot: experimental results from vapor-liquid equilibrium data [18]; Line: calculated results from Eq. (35). 


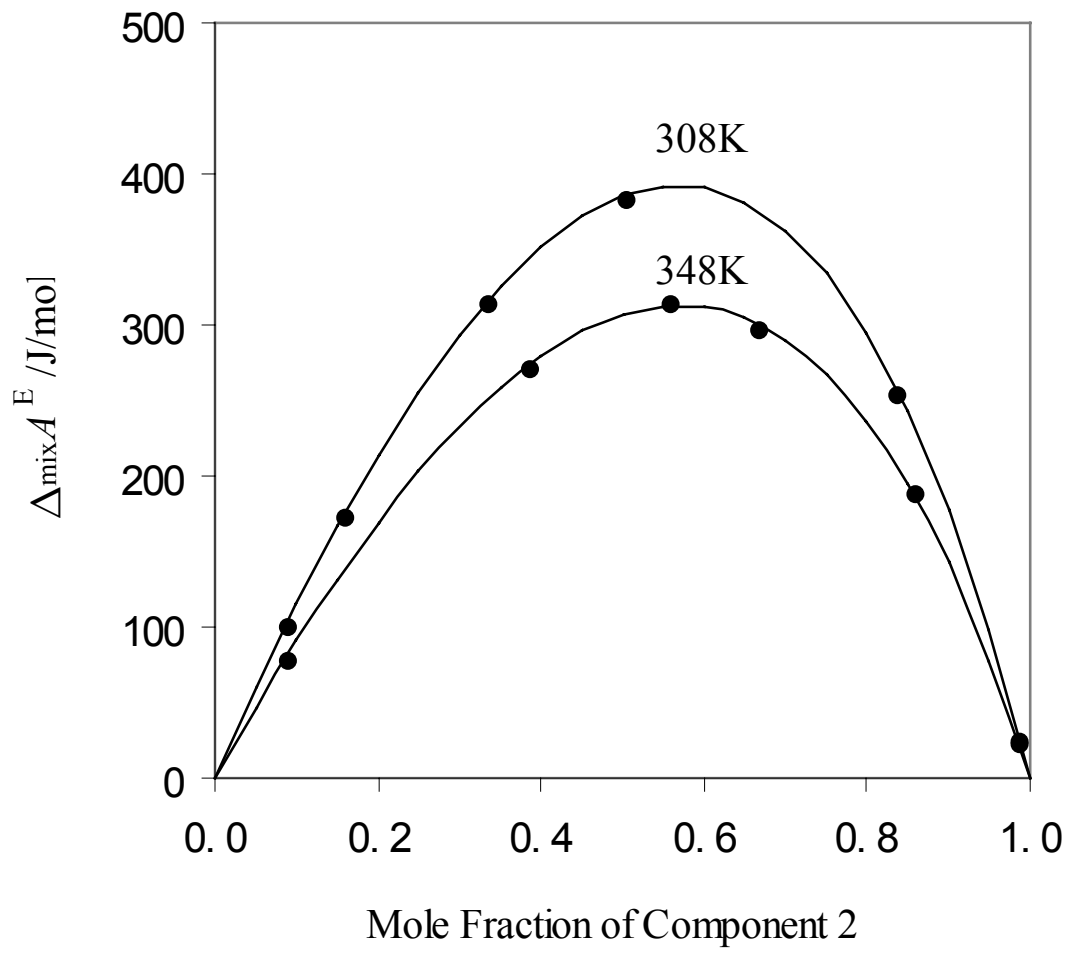

Fig. 6. Excess Helmholtz energy for the isooctane (1) / benzene (2) system at $308 \mathrm{~K}$ and $348 \mathrm{~K}$. Dot: experimental results from vapor-liquid equilibrium data [19]; Line: calculated results from Eq. (35). 


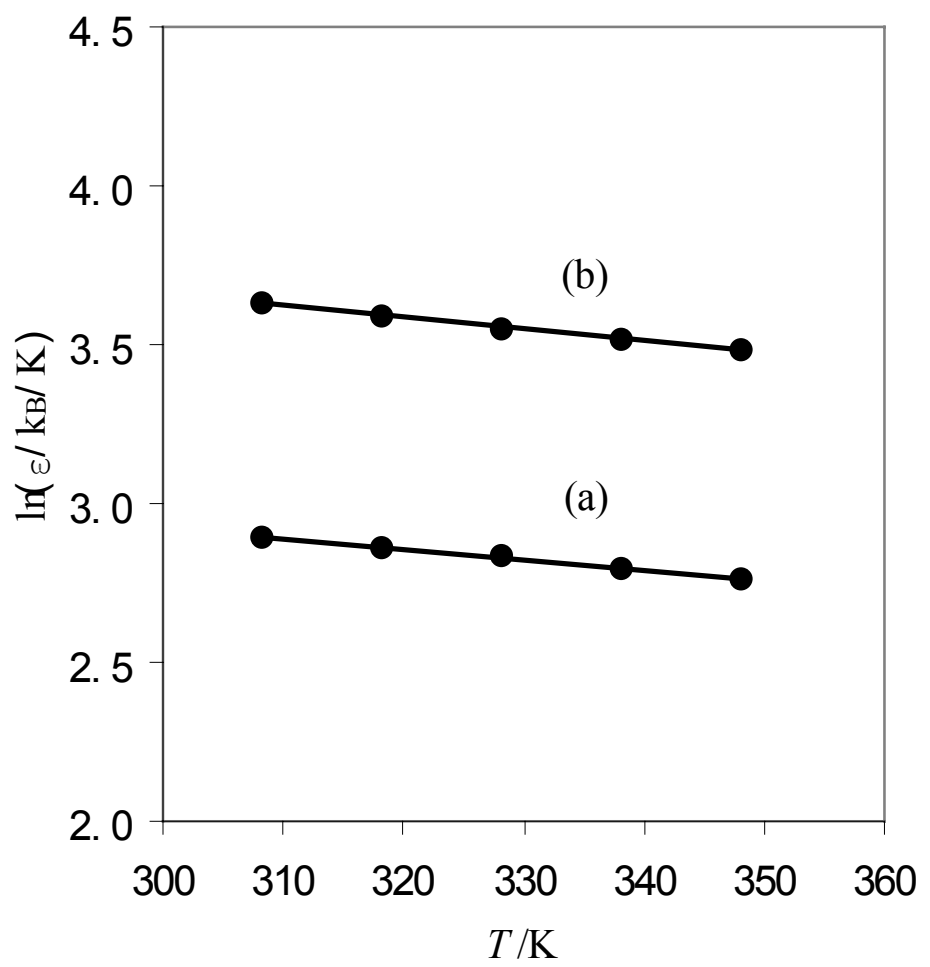

Fig. 7. Interchange energy $\varepsilon$ obtained from vapor-liquid equilibrium data vs. temperature. (a): isooctane / carbon tetrachloride; (b): isooctane / benzene. 


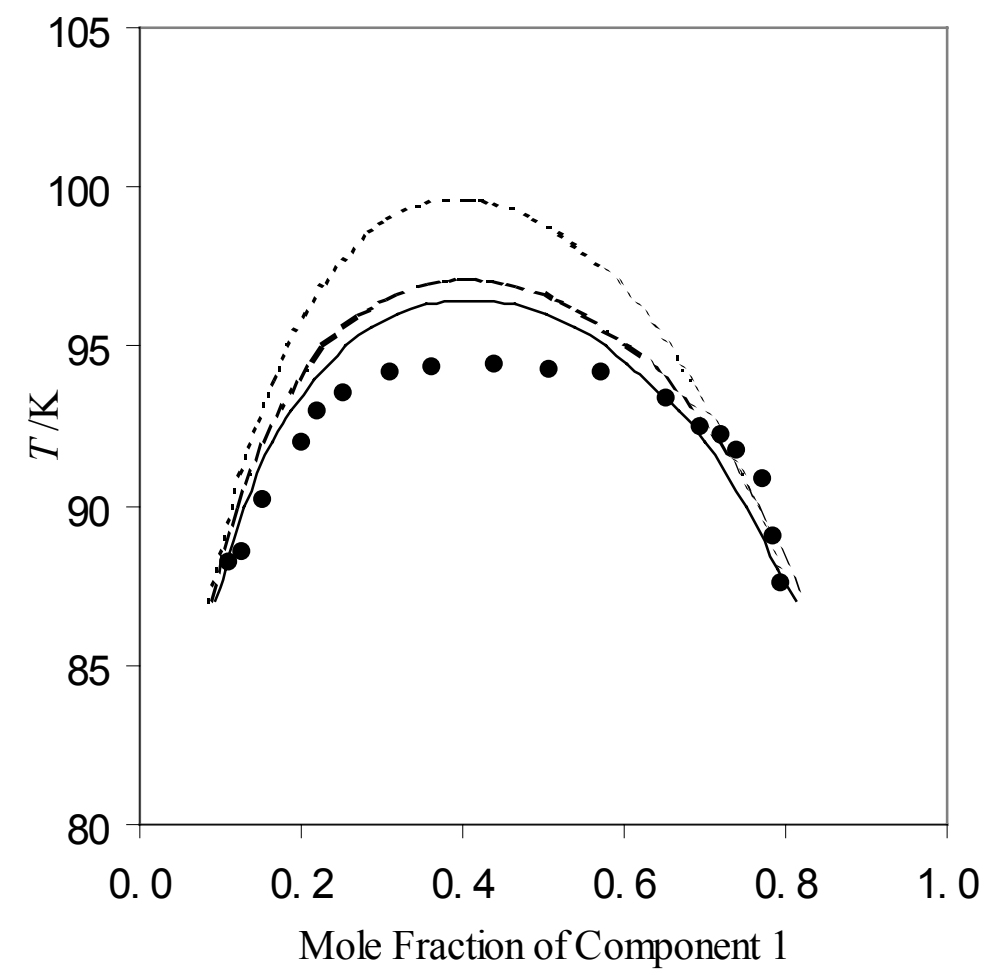

Fig. 8. Liquid-liquid equilibria for the $\mathrm{CF}_{4}$ (1) / $\mathrm{CH}_{4}$ (2) system. The coordination number $z=10 ; r_{1}=1.32, r_{2}=1.00$. Dot: Experimental data [21]; Dotted line: Flory-Huggins theory, $\ln \left(\varepsilon / k_{B} / K\right)=4.462-0.0091 T$; Dashed line: Quasi-chemical theory, $\ln \left(\varepsilon / k_{B} / K\right)=4.707$ $-0.0109 T$; Solid line: Eq. (35), $\ln \left(\varepsilon / k_{B} / K\right)=4.633-0.0101 T$. 


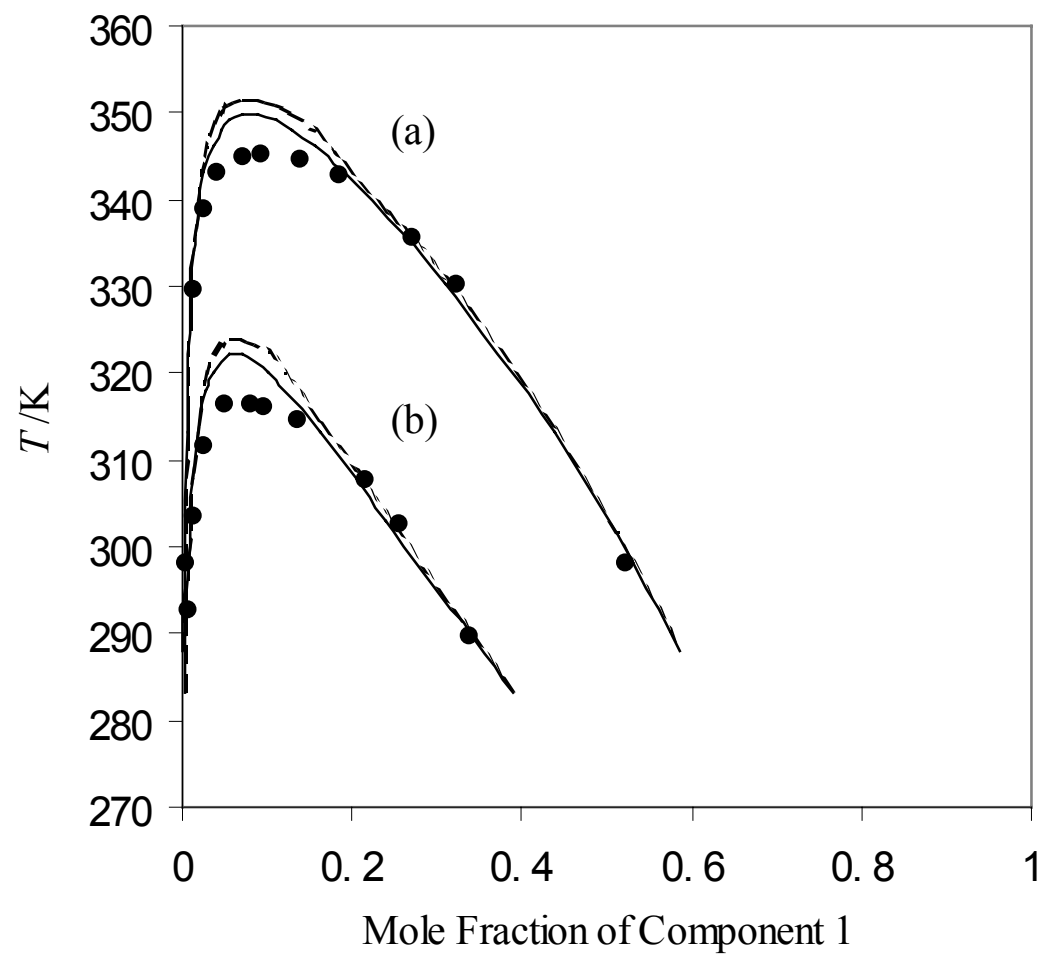

Fig. 9. Liquid-liquid equilibria for two binary systems. The coordination number $z=10$.

(a): $\left(\mathrm{C}_{3} \mathrm{~F}_{7} \mathrm{COOCH}_{2}\right)_{4} \mathrm{C}(1) / \mathrm{CHCl}_{3}(2)$ system. $r_{1}=6.77, r_{2}=1$. Solid line: Eq. (35), $\ln \left(\varepsilon / k_{B} / K\right)=6.02-0.00539 T$; Dotted line: quasi-chemical equation, $\ln \left(\varepsilon / k_{B} / K\right)=5.88$ $-0.00552 T$. (b): $\left(\mathrm{C}_{3} \mathrm{~F}_{7} \mathrm{COOCH}_{2}\right)_{4} \mathrm{C}$ (1) $/ \mathrm{CCl}_{4}(2)$ system. $r_{1}=5.61 ; r_{2}=1$. Solid line: Eq. (35), $\quad \ln \left(\varepsilon / k_{B} / K\right)=5.97-0.00432 T$. Dotted line: quasi-chemical equation, $\ln \left(\varepsilon / k_{B} / K\right)=5.69-0.00475 T$. These equations for $\ln \left(\varepsilon / k_{B} / K\right)$ were obtained from experimental LLE data well below the critical temperature. 